\title{
Strategic Interaction in Humans and Other Animals
}

\author{
Simon M. Huttegger · Brian Skyrms
}

Published online: 10 July 2013

(c) Konrad Lorenz Institute for Evolution and Cognition Research 2013

The history of game theory goes back to early 20th-century works by Emile Borel, Ernst Zermelo, and John von Neumann. But the field really took off with the publication of John von Neumann and Oskar Morgenstern's The Theory of Games and Economic Behavior in 1944. In the late '40s and during the '50s this led to a surge in interest for mathematical social sciences among mathematicians and economists. It was not until the ' 60 s and '70s that game theory started to play a role in biology. There is an early paper by Richard Lewontin on how classical game theory could be used in evolutionary biology (Lewontin 1961). At about the same time, William Hamilton introduced many game theoretic ideas such as the notion of an "unbeatable strategy" in Hamilton (1967). But the rebirth of game theory in biology is usually associated with John Maynard Smith and George Price's introduction of the concept of an "evolutionarily stable strategy" (Maynard Smith and Price 1973). Since then, evolutionary biologists and ecologists have used game theory as a tool to study the evolutionary dynamics of phenotypes. This development of biological game theory has merged more recently with a renewed interest in game theory in the social sciences and in economics. Contrary to classical game theory, which is mainly based on equilibrium analysis, this more recent approach puts games in the dynamic context of evolution and

S. M. Huttegger $(\bowtie) \cdot$ B. Skyrms

Department of Logic and Philosophy of Science,

University of California, Irvine, CA, USA

e-mail: shuttegg@uci.edu

B. Skyrms

e-mail: bskyrms@uci.edu

B. Skyrms

Department of Philosophy, Stanford University,

Stanford, CA, USA learning. While by no means exhausting the breadth and the depth of the field, the articles in this issue of Biological Theory allow a glimpse at some important questions of dynamic game theory within the social and biological sciences. The outlook is interdisciplinary, with contributions from biologists, economists, mathematicians, and philosophers.

The thematic issue starts off with Kevin Zollman's "Finding Alternatives to Handicap Theory." The handicap principle is an influential hypothesis in both biology and economics. It roughly states that communication is possible between a sender and a receiver who don't have completely common interests as long as the sender must pay a cost for sending signals. Zollman presents theoretical and empirical evidence against the handicap principle and presents possible alternatives to it that allow for communication in the face of conflicting interests.

Ted Bergstrom's paper develops an important topic in theoretical biology: the effect of correlation between types on evolution. Bergstrom discusses several approaches in his contribution, "Measures of Assortativity." The central concept is Wrights's F-statistic, which is a measure of inbreeding or, alternatively, relatedness. The paper compares the F-statistic to other measures of assortative matching. It also discusses the role of assortative matching in theories of altruistic behavior.

In "The Evolution of Simple Rule-Following," Jeffrey Barrett uses signaling games in the style of David Lewis to design models for transitive rule-following and analyzes them with the help of computer simulations. In addition, he considers models where agents can use already evolved or learned rules in new contexts. Agents in these models can learn to infer patterns and apply them to new contexts although they only learn by simple reinforcement learning schemes. 
Gene-culture coevolution has been a perennial approach in evolutionary game theory. In "Genes, Culture, and Preferences," Nikolaus Robalino and Arthur Robson apply this approach to the evolution of preferences. This is a particularly important topic for economics since it puts in place constraints on preferences. One particularly intriguing aspect of Robalino and Robson's paper is their outline of a theory of gene-culture coevolution that does not assume that genetic and environmental factors are independent.

One of the most important developments in game theory during the last decades was the rise of experimental economics. The article, "Game Experiments on Cooperation through Reward and Punishment" by Ross Cressman, Yi Tao, Jia-Jia $\mathrm{Wu}$, and Cong $\mathrm{Li}$, reports results on recent experiments about the effectiveness of punishment schemes in public goods games. Their findings point to the potential importance of cultural factors on the effect of punishment and to the influence of reward schemes when paired with punishment.

The research on evolutionary game theory uses advanced methods from the theory of dynamical systems. Visualization techniques often help to understand and also analyze models. Francisco Franchetti and Bill Sandholm offer an advanced approach to this in their contribution "An Introduction to Dynamo: Diagrams for Evolutionary Game Dynamics," in which they describe an open source software for evolutionary games together with a variety of applications.

Brian Skyrms, in "Natural Social Contracts," examines the foundations of a naturalistic social contract theory in terms of two well-known games, the Stag Hunt and the Nash bargaining game. Theoretical and experimental findings on these games are reviewed. The theoretical findings focus on dynamical approaches. This is based on the idea that the study of social interactions needs dynamics - an idea connecting together basically all the contributions to this thematic issue.
Rainer Hegselmann and Oliver Will describe the social contract along similar lines in "From Small Groups to Large Societies: How to Construct a Simulator?" The simulator tracks the evolution from small-scale to largescale populations, focusing on division of labor, risky exchange, moral control, and other issues. It is aptly named after David Hume, whose informal theory provides the background to the paper.

Information transfer-the topic of Zollman's and Barrett's contributions-is the motivation of Simon Huttegger's paper on "Probe and Adjust." Huttegger considers a very simple boundedly rational learning process that converges to the strict Nash equilibria in a certain class of games that comprises many relevant information transfer games.

Last, but not least, we have a contribution on "Sexual Drift" by Ken Binmore, who studies the effect of sexual reproduction on the evolutionary dynamics of an expanded Hawk-Dove game. This is not only interesting because it introduces sexual reproduction to game theoretic models, but also because it provides a mechanism for how populations can cross fitness valleys.

We hope that the reader will enjoy these articles as much as we do. They do not provide a survey of the work that is being done in evolutionary game theory. The reader gets served some highlights instead.

In the name of all the workshop participants, we wish to express our gratitude to the KLI for hosting our workshop and making this special issue possible.

\section{References}

Hamilton WD (1967) Extraordinary sex ratios. Science 156:477-488 Lewontin RC (1961) Evolution and the theory of games. J Theor Biol $1: 382-403$

Maynard Smith J, Price GR (1973) The logic of animal conflict. Nature 246:15-18 